\title{
176. On Abhomotopy Group in Relative Case
}

\author{
By Yoshiro INOUE \\ (Comm. by K. KUnUGI, M.J.A., Nov. 12, 1954)
}

\section{Introduction}

The $(n, r)$-th abhomotopy group $\kappa_{r}^{n}\left(Y, y_{0}\right)$ of a space $Y$ as base point $y_{0} \in Y$ was defined by S. T. Hu as a generalization of Abe groups (M. Abe [1]). He showed that its algebraic structure is completely determined in terms of homotopy groups of $Y$, and that

$$
\kappa_{r}^{n}\left(Y, y_{0}\right) \approx \pi_{r+1}\left(Y^{s^{n-r-1}}, k_{0}\right) \quad r \geq 0,
$$

where $Y^{s^{n-r-1}}$ is a mapping space consisting of all maps $f: S^{n-r-1} \rightarrow Y$ and topologized by compact open topology due to R.H.Fox (R. H. Fox [2]), and $k_{0}$ is a constant map: $k_{0}: S^{n-r-1} \rightarrow y_{0}$ (S. T. Hu[3]). In this paper, I shall show that the notion of abhomotopy group is relativized by using the same relation as $(*)$. In this paper, we always denote by $Y$ a given topological space, by $Y_{0}$ a subspace of $Y$ and $y_{0}$ a reference point of $Y_{0}$. Then the $(m, n)$-th relative abhomotopy group $\kappa_{n}^{m}\left(Y, Y_{0}, y_{0}\right)$ of $\left(Y, Y_{0}, y_{0}\right)$ is defined by

$$
\text { (**) } \quad \kappa_{n}^{m}\left(Y, Y_{0}, y_{0}\right)=\pi_{m}\left(Y^{E^{n}}\left\{S^{n-1}, Y_{0}\right\}, k_{0}\right) \quad m, n \geq 1,
$$

where $Y^{E^{n}}\left\{S^{n-1}, Y_{0}\right\}$ is a mapping space consisting of all maps $f$ : $E^{n}, S^{n-1} \rightarrow Y, Y_{0}$ and topologized by compact open topology. I shall show that, in $\S 2$, its algebraic structure is completely determined by $\pi_{m+n}\left(Y, Y_{0}, y_{0}\right)$ and $\pi_{m}\left(Y_{0}, y_{0}\right)$. In $\S 1$, for a preliminary of $\S 2$, I describe a definition of relative homotopy groups which is obtained by a slightly modification of that of absolute homotopy groups given in the book "S. T. Hu [4] 21 ".

§1. Preliminary. 1.1. Let $I^{n+1}$ be the $(n+1)$-cube, and $I^{n+1}$ be the boundary of $I^{n+1}$ as usual. We use the following notations:

$$
\begin{aligned}
& I^{n}=\left\{x^{n+1}=\left(x_{1}, \ldots, x_{n+1}\right) \in I^{n+1} \mid x_{n+1}=0\right\}, \\
& J^{n}=\check{I}^{n+1}-I^{n}, \\
& P_{n}^{n}=\left\{x^{n+1}=\left(x_{1}, \ldots, x_{n+1}\right) \in I^{n+1} \mid x_{n}=0\right\}, \\
& x_{0}=(0, \ldots, 0) \in \dot{I}^{n+1} .
\end{aligned}
$$

Let $\mathscr{F}=Y^{J^{n}}\left\{\dot{I}^{n}, Y_{0} ; x_{0}, y_{0}\right\}$ be the totality of maps $f: J^{n}, \dot{I}^{n}, x_{0}$ $\rightarrow Y, Y_{0}, y_{0}$. The maps $f$ of $\widetilde{F}$ are divided into disjoint homotopy classes relative to $\left\{\dot{I}^{n}, Y_{0} ; x_{0}, y_{0}\right\}$. Denote by $\Omega$ the totality of these classes and by $[f]$ the class containing $f \in \mathfrak{F}$. Let $f$ be a representative of an arbitrary element $\alpha$ of $\pi_{n}\left(Y, Y_{0}, y_{0}\right)$. Define a map $\mu f: J^{n} \rightarrow Y$ by taking for each $x^{n+1}=\left(x_{1}, \ldots, x_{n+1}\right) \in J^{n}$ 


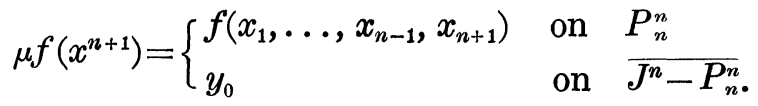

The map $\mu f$ belongs to $\mathfrak{F}$, and $[\mu f] \in \Omega$ depends only on the element $\alpha$. Then the correspondence $\alpha \rightarrow[\mu f]$ defines a one-to-one transformation $\mu^{*}: \pi_{n}\left(Y, Y_{0}, y_{0}\right) \rightarrow \Omega$ of $\pi_{n}\left(Y, Y_{0}, y_{0}\right)$ onto $\Omega$. The proofs of this fact and the following theorems are parallel to that given in "S. T. Hu [4] $\S 21$ ", and are omitted.

Theorem 1.1. For an arbitrary map $f \in \mathfrak{F},[f]=0$ if and only if $f$ has an extension $f^{*}: I^{n+1} \rightarrow Y$ such that $f^{*}\left(I^{n}\right) \subseteq Y_{0}$.

Theorem 1.2. Let $f, g \in \mathfrak{F}$ be two maps such that $\overline{f\left(\mathcal{J}^{m}-P_{n}^{n}\right)}=y_{0}$ $=g\left(P_{n}^{n}\right)$ and let $h \in \mathfrak{F}$ be the map defined by

$$
h(x)= \begin{cases}f(x) & x \in P_{n}^{n} \\ g(x) & x \in \overline{J^{n}-P_{n}^{n}}\end{cases}
$$

Then $[h]=[f]+[g]$.

1.2. It is well known that each element $\xi$ of $\pi_{1}\left(Y_{0}, y_{0}\right)$ induces an automorphism of $\pi_{n}\left(Y, Y_{0}, y_{0}\right)$, where $n \geq 2$ is any integer. Denote this automorphism by

$$
\xi^{*}: \alpha \rightarrow \alpha^{\xi} \quad \alpha \in \pi_{n}\left(Y, Y_{0}, y_{0}\right) .
$$

Let $\omega$ and $f$ be representatives of $\xi \in \pi_{1}\left(Y_{0}, y_{0}\right)$ and $\alpha \in \pi_{n}\left(Y, Y_{0}, y_{0}\right)$ respectively. Define a map $g: J^{n} \rightarrow Y$ by taking for each point $x=\left(x_{1}, \ldots, x_{n}, x_{n+1}\right) \in J^{n}$,

$$
g(x)=\left\{\begin{array}{lll}
f\left(x_{1}, \ldots, x_{n-1}, x_{n+1}\right) & \text { when } & x_{n}=1 \\
\omega\left(x_{n}\right) & \text { when } & 0<x_{n}<1 \\
y_{0} & \text { when } & x_{n}=0 .
\end{array}\right.
$$

The class $[g] \in \Omega$ depends only on $\alpha$ and $\xi$, and

$$
\alpha^{\xi}=-\mu^{*^{-1}}[g] \text {. }
$$

If, for every point $y_{0} \in Y_{0}$, the automorphisms $\xi^{*}$ defined above are always identical, the space $Y$ is called $n$-simple relative to $Y_{0}$.

§ 2. Relative Abhomotopy Groups. 2.1. Let $Y^{1^{n}}$ be a mapping space consisting of all maps $f: I^{n} \rightarrow Y$ and topologized by compact open topology, and let $\Re^{n}\left(y_{0}\right)$ be a subspace of the space $Y^{I^{n}}$, which consists of all maps $f: I^{n}, I^{n-1}, J^{n-1} \rightarrow Y, Y_{0}, y_{0}$, where $n \geq 1$ is any integer. Denote by $\mathfrak{F}^{n}\left(Y_{0}\right)$ the union of all $\mathfrak{F}^{n}(y)$ for $y \in Y_{0}$, i.e. $\mathfrak{F}^{n}\left(Y_{0}\right)=\breve{y \in Y}_{0} \widetilde{F}^{n}(y)$. Since $\widetilde{F}^{n}\left(y_{0}\right)$ is a subspace of the space $\mathfrak{F}^{n}\left(Y_{0}\right)$, we have the following homotopy sequence,

$$
\begin{aligned}
& \longrightarrow \pi_{m}\left(\mathfrak{F}^{n}\left(y_{0}\right), k_{0}\right) \stackrel{i_{m}}{\longrightarrow} \pi_{m}\left(\mathfrak{F}^{n}\left(Y_{0}\right), k_{0}\right) \stackrel{i_{m}}{\longrightarrow} \pi_{m}\left(\mho^{n}\left(Y_{0}\right), \mathfrak{F}^{n}\left(y_{0}\right), k_{0}\right) \\
& \stackrel{\partial_{m}}{\longrightarrow} \pi_{m-1}\left(\Re^{n}\left(y_{0}\right), k_{0}\right) \longrightarrow \cdots
\end{aligned}
$$


where $k_{0}$ is the constant map; $k_{0}: I^{n} \rightarrow y_{0}$. It is well known that

$$
\pi_{m}\left(\widetilde{\mho}^{n}\left(y_{0}\right), k_{0}\right) \approx \pi_{m+n}\left(Y, Y_{0}, y_{0}\right) .
$$

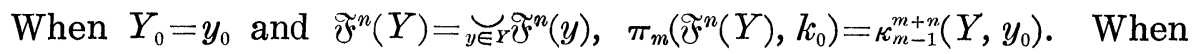
$m=1, \pi_{1}\left(\mathscr{F}^{n}\left(Y_{0}\right), k_{0}\right)$ identical with the group $\sigma_{n+1}\left(Y, Y_{0}, y_{0}\right)$ which was defined by $H$. Uehara in his paper [5]. We denote by $\kappa_{n}^{m}\left(Y, Y_{0}, y_{0}\right)$ the homotopy group $\pi_{m}\left(\widetilde{F}^{n}\left(Y_{0}\right), k_{0}\right)$ and call it the $(m, n)$ -th relative abhomotopy group of $\left(Y, Y_{0}, y_{0}\right)$. It is obvious that this definition is identical with $(* *)$ in the introduction. In the sequel, we shall study the algebraic structure of relative abhomotopy groups. First, we prove the following lemma.

Lemma 2.1. The image of the boundary homomorphism $\partial_{m}$ is only the neutral element, for every integer $m \geq 2$.

(proof) A representative $f$ of an arbitrary element $\alpha$ of $\pi_{m}\left(\widetilde{\mho}^{n}\left(Y_{0}\right), \mathfrak{F}^{n}\left(y_{0}\right), k_{0}\right)$ is characterized by

$$
f\left(x^{m}, x^{n}\right)=\left\{\begin{array}{lll}
y_{0} & \text { on } & J^{m-1} \times I^{n} \\
f\left(x_{1}, \ldots, x_{m-1}, 0, x^{n}\right) & \text { on } & I^{m-1} \times I^{n} \\
\omega\left(x^{m}\right) \in Y_{0} & \text { on } & I^{m} \times J^{n-1} \\
\in Y_{0} & \text { on } & I^{m} \times I^{n-1}
\end{array}\right.
$$

where $x^{m}=\left(x_{1}, \ldots, x_{m}\right) \in I^{m}, x^{n} \in I^{n}$. For this characterization, define the following two maps $g, h: \dot{I}^{m} \times I^{n} I^{m} \times J^{n-1}=J^{m+n-1} \rightarrow Y$ by taking

$$
\begin{aligned}
& g\left(x^{m}, x^{n}\right)=\left\{\begin{array}{lll}
f\left(x_{1}, \ldots, x_{m-1}, 0, x^{n}\right) & \text { on } & I^{m-1} \times I^{n} \\
y_{0} & \text { on } & J^{m-1} \times I^{n} \smile I^{m} \times J^{n-1}
\end{array}\right. \\
& h\left(x^{m}, x^{n}\right)=\left\{\begin{array}{lll}
\omega\left(x^{m}\right) & \text { on } & I^{m} \times J^{n-1} \\
y_{0} & \text { on } & I^{m-1} \times I^{n} \smile J^{m-1} \times I^{n} .
\end{array}\right.
\end{aligned}
$$

The maps $g$ and $h$ represent the element $\beta, \gamma \in \pi_{m+n}\left(Y, Y_{0}, y_{0}\right)$ respectively. From the definition, $\beta=\partial_{m} \alpha$. Let $f_{0}$ be the partial map : $f_{0}=f \mid \dot{I}^{m} \times I^{n} \smile I^{m} \times J^{n-1}$. Then by Theorem 1.2, $\left[f_{0}\right]=[g]+[h]$. Since $f_{0}$ has an extension $f$ to $I^{m} \times I^{n}$, and since $h$ has an extension $h^{*}: I^{m} \times I^{n} \rightarrow Y$ such that

$$
h^{*}\left(x^{m}, x^{n}\right)=h\left(x^{m}\right) \quad \text { on } \quad I^{m} \times I^{n},
$$

then $\left[f_{0}\right]=[h]=0$ by Theorem 1.1. Hence $[g]=0$, i.e. $\beta=\partial \alpha=0$. This completes the proof.

By the lemma stated above and from the exactness of the homotopy sequence (1), the homomorphism $i_{m}$ is isomorphic into and the homomorphism $j_{m}$ is onto. Therefore, the group $\kappa_{n}^{m}\left(Y, Y_{0}, y_{0}\right)$ contains a normal subgroup $\bar{\pi}_{m+n}$ isomorphic to $\pi_{m+n}\left(Y, Y_{0}, y_{0}\right)$.

A representative $f$ of an arbitrary element $\alpha$ of $\kappa_{n}^{m}\left(Y, Y_{0}, y_{0}\right)$ is characterized by 


$$
f\left(x^{m}, x^{n}\right)=\left\{\begin{array}{lll}
y_{0} & \text { on } & \dot{I}^{m} \times I^{n} \\
\omega\left(x^{m}\right) \in Y_{0} & \text { on } & I^{m} \times J^{n-1} \\
\in Y_{0} & \text { on } & I^{m} \times I^{n-1},
\end{array}\right.
$$

where $\omega\left(x^{m}\right)=f\left(x^{m}, 0, \ldots, 0\right)$. It is clear that $\omega\left(x^{m}\right) \in Y^{r^{m}}\left\{\dot{I}^{m}, y_{0}\right\}$. The element $\beta$ of $\pi_{m}\left(Y_{0}, y_{0}\right)$ represented by $\omega$ depends only on $\alpha$. By making correspondence $\alpha$ to $\beta$, we obtain a homomorphism:

$$
p^{*}: \kappa_{n}^{m}\left(Y, Y_{0}, y_{0}\right) \rightarrow \pi_{m}\left(Y_{0}, y_{0}\right) \text {. }
$$

Conversely, for a representative $\omega$ of an element $\beta \in \pi_{m}\left(Y_{0}, y_{0}\right)$, the map $f_{\omega}: I^{m} \times I^{n} \rightarrow Y$ defined by

$$
f_{\omega}\left(x^{m}, x^{n}\right)=\omega\left(x^{n}\right) \quad \text { on } \quad I^{m} \times I^{n}
$$

is a representative of an element $\alpha_{\omega}$ of $\kappa_{n}^{m}\left(Y, Y_{0}, y_{0}\right)$ and $p^{*}\left(\alpha_{\omega}\right)=\beta$. The totality of such elements constructs a subgroup $\bar{\pi}_{m}$ of $\kappa_{n}^{m}\left(Y, Y_{0}, y_{0}\right)$ isomorphic to $\pi_{m}\left(Y_{0}, y_{0}\right)$. Therefore $p^{*}$ is onto.

Lemma 2.2. Kernel $p^{*}=$ image $i_{m}^{*}$, for every integer $m \geq 1$.

(proof) It is clear that $p^{*} i_{m}^{*}=0$, conversely, we suppose that $p^{*} \alpha=0$ for an element $\alpha \in \kappa_{n}^{m}\left(Y, Y_{0}, y\right)$. A representative $f$ of $\alpha$ is characterized by (4). From the assumption $p^{*} \alpha=0$, there exists a homotopy $\omega_{t}: I^{m} \rightarrow Y(0 \leq t \leq 1)$ such that $\omega_{0}=\omega, \omega_{1}=y_{0}$. Define a homotopy $h_{t}: J^{m+n-1}=\dot{I}^{m} \times \dot{I}^{n} \smile I^{m} \times J^{n-1} \rightarrow Y(0 \leq t \leq 1)$ by

$$
h_{t}\left(x^{m}, x^{n}\right)=\left\{\begin{array}{lll}
y_{0} & \text { on } & \dot{I}^{m} \times I^{n} \\
\omega_{t}\left(x^{m}\right) & \text { on } & I^{m} \times J^{n-1}
\end{array} .\right.
$$

The homotopy $h_{t}$ has an extension $h_{t}^{*}: I^{m} \times I^{n} \rightarrow Y$ such that $h_{0}^{*}=f$, $h_{1}^{*}\left(J^{m+n-1}\right)=y_{0}$, and $h_{t}^{*}\left(I^{m+n-1}\right) \subseteq Y_{0}$. Obviously, the map $h_{1}^{*}$ is a representative of an element $\gamma \in \pi_{m}\left(\mathscr{\mho}^{n}\left(y_{0}\right), k_{0}\right)$. By the homotopy $h_{t}^{*}$, $i_{m}^{*} \gamma=\alpha$. This completes the proof.

By Lemmas 2.1 and 2.2, and from the exactness of the homotopy sequence (1), we have an isomorphism:

$$
\pi_{m}\left(\widetilde{\mho}^{n}\left(Y_{0}\right), \widetilde{\mho}^{n}\left(y_{0}\right), k_{0}\right) \approx \kappa_{n}^{m}\left(Y, Y_{0}, y_{0}\right) / \bar{\pi}_{m+n} \approx \pi_{m}\left(Y_{0}, y_{0}\right) .
$$

Summalizing, from the commutativity of the group $\kappa_{n}^{m}\left(Y, Y_{0}, y_{0}\right)$ for $m \geq 2$, we have the following theorem.

Theorem 2.3. The group $\kappa_{n}^{m}\left(Y, Y_{0}, y_{0}\right)(m \geq 1, n \geq 1)$ contains a normal subgroup $\bar{\pi}_{m+n}$ isomorphic to $\pi_{m+n}\left(Y, Y_{0}, y_{0}\right)$ and a subgroup $\bar{\pi}_{m}$ isomorphic to $\pi_{m}\left(Y_{0}, y_{0}\right)$. When $m \geq 2, \kappa_{n}^{m}\left(Y, Y_{0}, y_{0}\right)$ decomposes into the direct sum of two subgroups $\bar{\pi}_{m+n}$ and $\bar{\pi}_{m}$ :

$$
\kappa_{n}^{m}\left(Y, Y_{0}, y_{0}\right)=\bar{\pi}_{m+n}+\bar{\pi}_{m} \approx \pi_{m+n}\left(Y, Y_{0}, y_{0}\right)+\pi_{m}\left(Y_{0}, y_{0}\right) .
$$

2.2. When $m=1$, the group $\kappa_{n}^{1}\left(Y, Y_{0}, y_{0}\right)$ is a generalization of Abe groups. The group $\kappa_{n}^{1}\left(Y, Y_{0}, y_{0}\right)$ contains a normal subgroup $\bar{\pi}_{n+1}$ isomorphic to $\pi_{n+1}\left(Y, Y_{0}, y_{0}\right)$ and a subgroup $\bar{\pi}_{1}$ isomorphic to 
$\pi_{1}\left(Y_{0}, y_{0}\right)$. In the group $\kappa_{n}^{1}\left(Y, Y_{0}, y_{0}\right)$, the operation of $\pi_{1}\left(Y_{0}, y_{0}\right)$ on $\pi_{n+1}\left(Y, Y_{0}, y_{0}\right)$ induces an inner automorphism:

$$
\alpha^{\xi}=\bar{\xi} \alpha \bar{\xi}^{-1},
$$

where $\bar{\xi}$ is the element of $\pi_{1}$ such that $p^{*}(\bar{\xi})=\xi$.

We prove the relation (7). From the definition of $\alpha^{\xi}$, two maps $f, g$ representing $\alpha$ and $\alpha^{\xi}$ are free homotopic relative to $Y_{0}$ with respect to the path $\omega$ representing $\xi$. Then there exists a map $F: I^{n+1} \times I \rightarrow Y$ such that

$$
\begin{array}{ll}
F\left(x^{n+1}, 1\right)=f\left(x^{n+1}\right), & F\left(x^{n+1}, 0\right)=g\left(x^{n}\right) \\
F\left(J^{n}, t\right)=\omega(t), & F\left(I^{n}, t\right) \subseteq Y_{0} .
\end{array}
$$

Define a homotopy $h_{s}: I^{n+1} \rightarrow Y(0 \leq s \leq 1)$ by

$$
h_{s}\left(x^{n+1}\right)= \begin{cases}F\left(0, x_{2}, \ldots, x_{n+1}, 3 x_{1}\right)=\omega\left(3 x_{1}\right) & 0 \leq x_{1} \leq \frac{1}{3} s \\ F\left(\frac{3 x_{1}-s}{3-2 s}, x_{2}, \ldots, x_{n+1}, s\right) & \frac{1}{3} s \leq x_{1} \leq 1-\frac{1}{3} s \\ F\left(1, x_{2}, \ldots, x_{n+1}, 3-3 x_{1}\right)=\omega\left(3-3 x_{1}\right) & 1-\frac{1}{3} s \leq x \leq 1 .\end{cases}
$$

Then $h_{0}=g$ and $\left[h_{1}\right]=\bar{\xi} \alpha \bar{\xi}^{-1}$. By the homotopy $h_{s}, \alpha^{\xi}=\bar{\xi} \alpha \bar{\xi}^{-1}$. This establishes the relation (7). Thus, we have the following result.

Theorem 2.4. The group $\kappa_{n}^{1}\left(Y, Y_{0}, y_{0}\right)(n \geq 1)$ contains a normal subgroup $\bar{\pi}_{n+1}$ isomorphic to $\pi_{n+1}\left(Y, Y_{0}, y_{0}\right)$ and a subgroup $\bar{\pi}_{1}$ isomorphic to $\pi_{1}\left(Y_{0}, y_{0}\right)$, and is a split extention of $\pi_{n+1}\left(Y, Y_{0}, y_{0}\right)$ by $\pi_{1}\left(Y_{0}, y_{0}\right)$. A necessary and sufficient condition for $Y$ to be $(n+1)$ simple relative to $Y_{0}$ is that $\kappa_{n}^{1}\left(Y, Y_{0}, y_{0}\right)$ decomposes into the direct product:

$$
\kappa_{n}^{1}\left(Y, Y_{0}, y_{0}\right)=\bar{\pi}_{n+1} \times \bar{\pi}_{1} \approx_{\pi_{n+1}}\left(Y, Y_{0}, y_{0}\right) \times \pi_{1}\left(Y_{0}, y_{0}\right)
$$

\section{References}

[1] M. Abe: Über die stetigen Abbildungen der $n$-Sphäre in einen metrischen Raum, Jap. J. Math., 16, 169-176 (1940).

[2] R. H. Fox: On topologies for function spaces, Bull. Amer. Math. Soc., 51, 429-432 (1945).

[3] S. T. Hu: On spherical mapping in a metric space, Ann. Math., 48, 717-734 (1947).

[4] - : Homotopy Theory, 1, Tulane University (1950).

[5] H. Uehara: Some remarks on relative free homotopy, Jap. J. Math., 2, 247-252 (1951). 